\title{
Postural Dynamics and Vocal Effort: Preliminary Experimental Analysis
}

\author{
Antoine Giovanni $^{\mathrm{a}} \quad$ Leslie Akl $^{\mathrm{b}} \quad$ Maurice Ouaknine $^{\mathrm{a}}$ \\ a'Laboratoire d'Audio-Phonologie Expérimentale et Clinique de l'Université de la Méditerranée, Marseille, France; \\ bSaint Joseph University, Institut Supérieur d'Orthophonie, Beirut, Lebanon
}

\section{Key Words \\ Vocal effort • Postural activity · Force platform}

\begin{abstract}
Objectives: One postural characteristic of increased vocal effort is stiffening of the whole body, which in turn induces some postural instability. The study of postural instability can shed some insight into behavior or causes associated with vocal hyperfunction. Patients and Methods: A posturography platform was used to measure the root mean square of the velocity of variation of the center of pressure (VCOP rms) of control subjects standing upright on the platform. Four conditions were analyzed: (1) standing with eyes open versus eyes closed (n: 9); (2) standing at rest versus holding a 3-kg weight (n: 15); (3) standing while reading a dialogue at normal voice level versus loud voice (n: 17), and (4) standing while reading a dialogue in a calm versus noisy (Lombard effect) ambiance (n: 19). Results: Conditions leading to a stiffening of the body (eyes closed and weight holding) were associated with an increased instability assessed by the VCOP rms. In phonatory conditions leading to vocal effort, the mean VCOP rms was significantly increased: in 19 subjects reading a dialogue in a calm ambiance the mean VCOP rms was at $11.19 \mathrm{~mm} / \mathrm{s}$, and the VCOP rms raised 16.69 $\mathrm{mm} / \mathrm{s}$ with surrounding noise at $82 \mathrm{db}$ (Lombard effect). Conclusion: Increased vocal effort is associated with in-
\end{abstract}

\section{KARGER \\ Fax +41613061234 \\ E-Mail karger@karger.ch}

www.karger.com (c) 2008 S. Karger AG, Basel

$1021-7762 / 08 / 0602-0080 \$ 24.50 / 0$

Accessible online at:

www.karger.com/fpl creased VCOP rms in most subjects studied. The effects of VCOP rms as a postural index allowing noninvasive assessment of the voice in pathology warrants further study.

Copyright $\odot 2008$ S. Karger AG, Basel

\section{Introduction}

Speech therapy is used to treat muscle tension dysphonia and, more generally speaking, hyperfunctional dysphonia. Various techniques can be effective, including respiration control, relaxation and vocal exercises as well as exercises to improve control of postural dynamics. Many studies have focused on respiration control, relaxation and vocal exercises but there is little data on posture and its relation to vocal effort [1].

The prototypical postural features of hyperfunctional dysphonia are anterior projection of the head and shoulders and increased tension in the neck, leg and back muscles $[2,3]$. The same features can be observed when normal subjects shout to attract attention or be heard in a noisy environment [3]. Clinical assessment of postural changes could provide useful information but requires relatively complicated devices such as kinematic systems. Based on the assumption that postural adjustments affect the balance of the entire body, it has been hypothesized that a force platform might be a useful tool [4].
Antoine Giovanni

Fédération ORL CHU Timone

FR-13385 Marseille Cedex 5 (France)

Tel. +334913860 69, Fax +33481387454

E-Mail antoine.giovanni@ap-hm.fr 
Force platforms were developed to measure ground forces applied by the feet and to compute the center of pressure (COP) [5-7]. Once the COP coordinates have been determined, the velocity of body sway can be easily calculated $[8,9]$. Force platforms have often been used to study equilibrium strategies especially in subjects with vertigo and postural instability [10]. Postural stability is defined as the ability to maintain and control the body center within the base of support so as to prevent falling and complete desired movements. Control of postural stability involves intricate neuromuscular mechanisms using proprioceptive, vestibular and visual input.

Previously we presented a pilot study using a force platform to compare postural behavior in patients and controls [4]. The control group included 16 subjects with normal voices and no history of laryngeal disease (mean age, 27 years). The patient group included 26 subjects with vocal nodules (mean age, 30 years). The test task consisted of reading a declarative sentence loudly and forcefully. During the task variation of COP was measured and the ratio between variance in velocity of swaying during phonation and at rest (without phonation) was calculated. The findings showed a significant difference between patients and controls: 0.32 versus 0.07 . These findings suggest that phonatory activity in patients with vocal hyperfunction involved postural instability. The term phonoposturology was coined to designate the clinical and physiopathological study of this phenomenon.

The purpose of this experimental study was to validate postural measures obtained using a force platform for the assessment of vocal effort during various conditions of phonation in healthy control subjects.

\section{Methods}

\section{Subjects}

A total of 60 healthy women with a mean age of 25 (range, 21-28) were recruited. The decision to test young females was made because a nursing school was located nearby the laboratory. Different subjects were enrolled for each of the 4 experimental protocols described below.

\section{Methodology}

All protocols were carried out using a force platform designed in our laboratory. The main components of the platform are as follows:

- $55 \times 55 \mathrm{~cm}$ ground platform;

- 3 strength gauge transducers (Médicapteurs, Toulouse, France) allowing accuracy in the order of $20 \mathrm{~g}$ for weight and \pm 0.1 $\mathrm{mm}$ for COP;

- data acquisition card (Médicapteurs) with a band width from 0 to $500 \mathrm{~Hz}$, precision $16 \mathrm{bits} / 100 \mathrm{~kg}$.
All platform recordings lasted $51.2 \mathrm{~s}$ as per the recommendations of the French Association of Posturology. The final index used for all 4 protocols was the root mean square of the velocity of displacement from the COP (VCOP rms) in millimeters/second.

\section{Protocol 1}

It is well documented that standing upright requires more postural muscle activity (stiffening) with eyes closed than eyes open, the most likely explanation being absence of visual input $[11,12]$. Protocol 1 was designed to show that posturographic measurements were able to detect increased muscle activity in subjects standing with eyes closed and that the findings could be correlated with electromyographic (EMG) activity in the leg muscles, using the soleus muscle as the gold standard. The working hypothesis was that stiffening while standing upright with eyes closed would lead to a simultaneous increase in VCOP rms and soleus muscle activity.

Measurements of VCOP rms and EMG activity in the soleus muscle were made in 9 normal subjects. Each subject was first asked to stand motionless on the platform for $51.2 \mathrm{~s}$ while looking straight ahead with eyes open (EO) (condition 1). After a few minutes of rest, each subject was asked to repeat the task for the same length of time with eyes closed (EC) (condition 2). The EMG signal was recorded using dry disposable surface $\mathrm{Ag}$ - $\mathrm{AgCl}$ electrodes (Comepa, Paris, France). The signal was amplified by a single amplifying device built in our laboratory (noise, $<10 \mu \mathrm{V}$ ), rectified and low-band filtered $(100 \mathrm{~Hz})$.

For statistical analysis we determined the standard deviation (SD) of the EMG signal amplitude. We used the normalized value calculated with the standard formula, i.e. (condition 1 - condition 2)/(condition $1+$ condition 2). In the present study the normalized value was obtained by dividing the ratio of the differences between SD in EO and EC conditions by the sum of values in EO and $\mathrm{EC}$ conditions. The result is a percentage. We also measured VCOP rms as described above. Comparisons of the normalized differences in VCOP rms values and EMG activity observed under EO and EC conditions were performed using the Pearson nonparametric correlation test. The results were expressed in $r^{2}$ that was considered as the coefficient of determination corresponding to common variance.

\section{Protocol 2}

Protocol 2 was designed to compare VCOP rms values in normal subjects standing empty-handed (condition 1) or holding weights (condition 2). Since weight holding requires body stiffening, the working hypothesis was the same as in the previous protocol, i.e. that condition 2 would be associated with significantly higher VCOP rms.

Measurements of VCOP rms were made in 15 normal subjects. Each subject was asked to stand upright on the platform for $51.2 \mathrm{~s}$ first holding nothing in the hands and then, after a period of rest, holding a 3-kg weight for the same length of time.

The objective of statistical analysis was to demonstrate a difference between the 2 experimental conditions. The nonparametric Mann-Whitney test was used, since normal distribution of the measured values seemed unlikely. 
Protocol 3

The goal of protocol 3 was to demonstrate an increase in VCOP rms during loud speech. The working hypothesis was that muscle stiffening would increase as a result of higher energy requirements associated with loud speech.

Measurements of VCOP rms were made in 17 normal subjects. Each subject was asked to read a standardized text in the form of a polite 4-line dialogue with another person. The dialogue was performed twice, i.e. first at a normal volume understandable to the other person at a distance of $1.5 \mathrm{~m}$ (condition 1 ) and second at a loud volume understandable to the other person at a distance of $4.0 \mathrm{~m}$ (condition 2). To preserve conversational spontaneity, no attempt was made to standardize voice intensity during either reading but the experimental observer verified that all subjects increased voice intensity during the second reading. As in the other protocols the experiment lasted $51.2 \mathrm{~s}$. VCOP rms was the mean of the 4 values measured during each line of the dialogue.

Both readings were recorded with a microphone (Behringer, Germany) mounted on a vibration-free holder. The vocal signal was then amplified and digitalized with the same multichannel acquisition card as the other signals. Because appropriate calibration was not performed, the actual intensity could not be determined. All signals were synchronously displayed with a software program written in our laboratory (Signal Lab ${ }^{\circledR}$, Marseille, France). The general layout of the experience is shown in figure 1.

The data obtained in the 2 experimental conditions were compared using the Student test for paired values.

\section{Protocol 4}

In the fourth protocol variations in VCOP rms were analyzed under Lombard conditions. The Lombard effect is the natural tendency of a subject to increase vocal intensity in response to background noise or poor acoustics. The classical explanation of the Lombard effect involves a physiological reflex but it could also correspond to a behavioral strategy aimed at maintaining the quality of the communication. As stated by Lane and Tranel [13], 'the speaker does not change his voice level to communicate better with himself but rather with the others'. Several studies on voice forcing have been based on this aspect of the voice physiology $[14,15]$.

Measurements of VCOP rms were made in 19 normal subjects. The task involved 2 readings of the same dialogue as in protocol 3 with another person standing at $1.5 \mathrm{~m}$. The first reading was done with a calm ambiance (experimental condition 1) and the second under Lombard condition achieved by playing a 'cocktail party' soundtrack at $90 \mathrm{db}$ through a loudspeaker located $1.5 \mathrm{~m}$ from the subject. The soundtrack was taken from the acoustic files of the Laboratoire d'Acoustique Musicale de Paris. The level of $90 \mathrm{db}$ was set based on preliminary experiments.

The data obtained in the 2 experimental conditions were compared using the Student test for paired values.

\section{Results}

Protocol 1 data are shown in table 1 . The EMG values correspond to the normalized difference between activity observed under EO and EC. The VCOP rms values

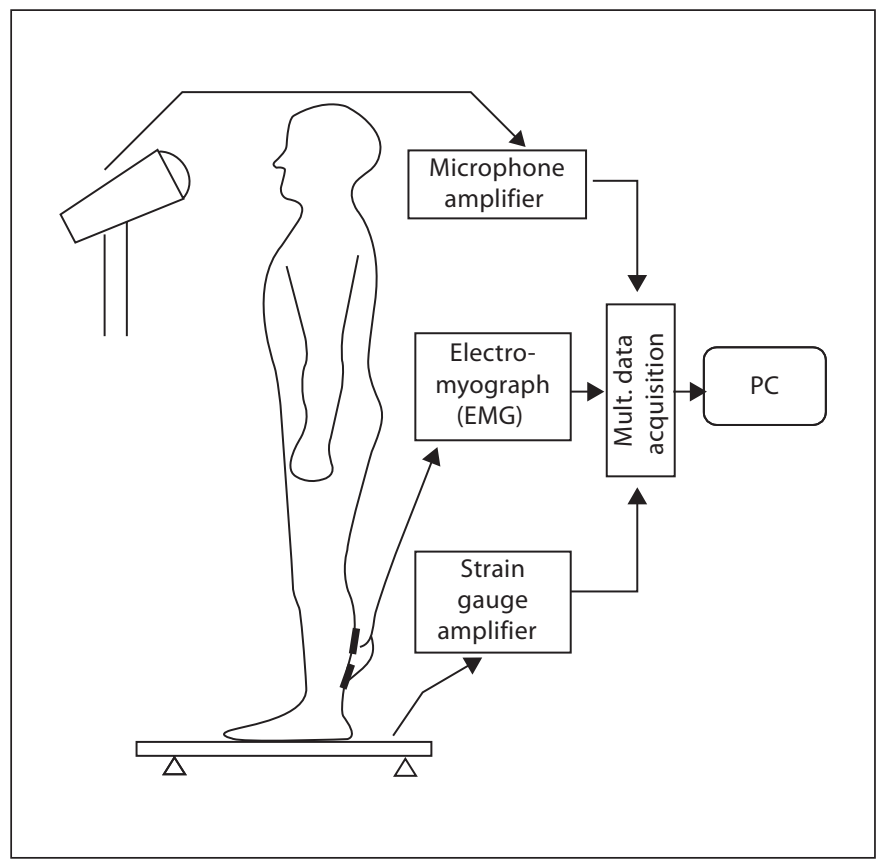

Fig. 1. General experimental layout of protocol 3.

Table 1. Comparison of EMG signal (normalized difference of values of EMG activity and VCOP rms in millimeters/second) in the 2 experimental conditions

\begin{tabular}{llcll}
\hline Subject & $\begin{array}{l}\text { VCOP rms } \\
\text { eyes open }\end{array}$ & $\begin{array}{l}\text { VCOP rms } \\
\text { eyes closed }\end{array}$ & $\begin{array}{l}\text { Normalized } \\
\text { difference, \% }\end{array}$ & $\begin{array}{l}\text { EMG } \\
\%\end{array}$ \\
\hline 1 & 6.49 & 12.72 & 32 & 31 \\
2 & 8.13 & 12.84 & 22 & 19 \\
3 & 8.85 & 17.32 & 32 & 47 \\
4 & 9.73 & 16.99 & 27 & 22 \\
5 & 8.11 & 9.32 & 7 & 13 \\
6 & 6.84 & 10.24 & 20 & 13 \\
7 & 8.74 & 12.97 & 19 & 9 \\
8 & 6.72 & 8.89 & 13 & 4 \\
9 & 5.93 & 11.08 & 30 & 29 \\
Mean & 7.73 & 12.49 & & \\
SD & 1.28 & 3.04 & & \\
\hline
\end{tabular}

Normalized difference in percent was calculated using the formula: (condition 1 - condition 2$) /($ condition $1+$ condition 2$)$.

measured under EO and EC conditions are also listed. In all subjects, an increase in VCOP rms and soleus muscle activity was observed under EC conditions. Despite the small size of the population, the difference in VCOP rms values under EO and EC conditions was 


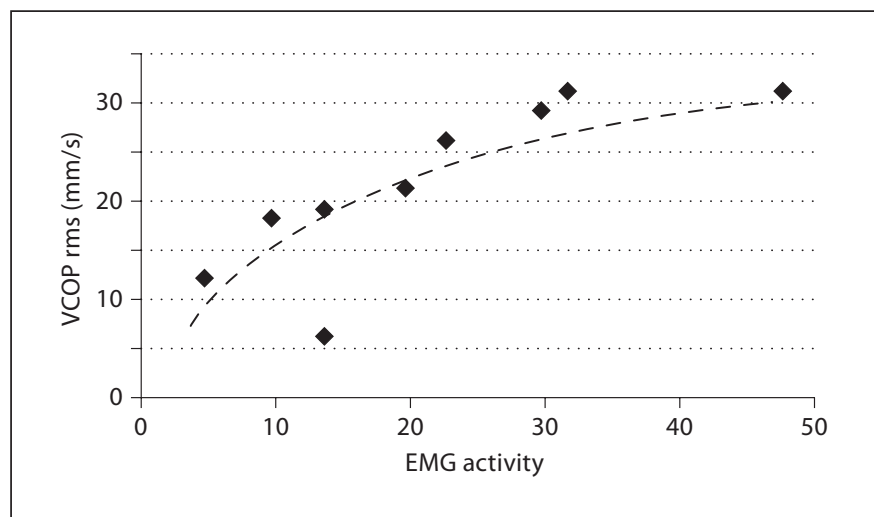

Fig. 2. Graph showing correlation between normalized values of $\mathrm{SD}$ of EMG signal (see text) on $\mathrm{x}$-axis and VCOP rms on $\mathrm{y}$-axis.

Table 2. Protocol 2: VCOP rms values (in millimeters/second) in a function of weight holding

\begin{tabular}{lcc}
\hline Subject & Condition 1 & Condition 2 \\
\hline 1 & 6.08 & 10.88 \\
2 & 6.53 & 12.64 \\
3 & 6.32 & 14.72 \\
4 & 7.20 & 11.52 \\
5 & 6.95 & 12.86 \\
6 & 5.12 & 12.00 \\
7 & 9.73 & 19.04 \\
8 & 6.88 & 11.40 \\
9 & 6.88 & 11.32 \\
10 & 12.48 & 21.15 \\
11 & 7.68 & 12.86 \\
12 & 4.32 & 8.96 \\
13 & 10.96 & 12.64 \\
14 & 4.65 & 7.04 \\
15 & 7.04 & 12.64 \\
Mean & 7.25 & 12.77 \\
SD & 2.24 & 3.48 \\
\hline
\end{tabular}

Condition 1: empty-handed. Condition 2: holding a 3-kg weight.

statistically significant $(\mathrm{p}<0.001)$ with a mean of $7.8 \mathrm{~mm} / \mathrm{s}$ (SD: 1.28 ) and $12.04 \mathrm{~mm} / \mathrm{s}$ (SD: 3.04 ) respectively. Analysis also demonstrated good correlation between VCOP rms and EMG signal variation (fig. 2) with a coefficient of determination $\mathrm{r} 2$ (common variance) of $64 \%$.

Data from protocol 2 designed to specifically study the effects of postural muscle stiffening are listed in table 2.

Postural Dynamics

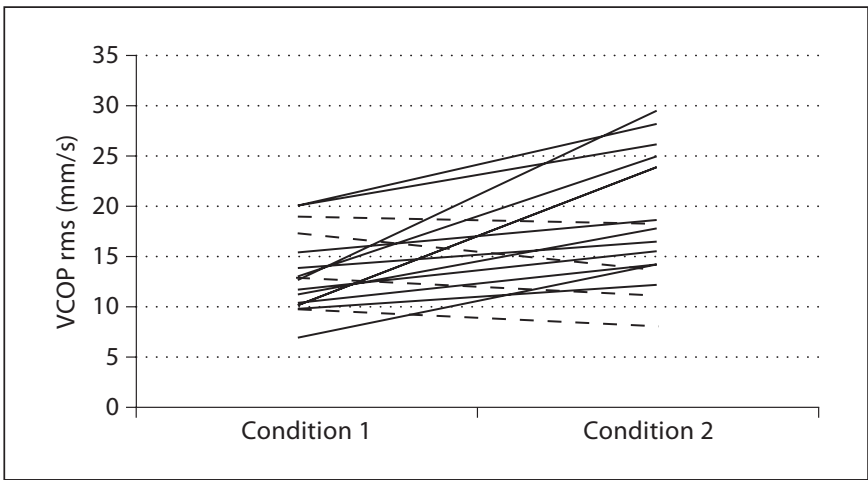

Fig. 3. Protocol 3: VCOP rms on y-axis. Condition 1: reading dialogue at $1 \mathrm{~m}$. Condition 2: reading dialogue at $4 \mathrm{~m}$. Stippled lines correspond to discordances with working hypothesis.

Table 3. Protocol 3: VCOP rms (in millimeters/second) values in a function of speech volume

\begin{tabular}{lcc}
\hline Subject & Condition 1 & Condition 3 \\
\hline 1 & 9.76 & 12.24 \\
2 & 13.80 & 16.48 \\
$3^{\mathrm{a}}$ & 9.85 & 8.16 \\
4 & 11.20 & 17.80 \\
5 & 13.05 & 24.96 \\
$6^{\mathrm{a}}$ & 19.04 & 18.25 \\
7 & 12.64 & 29.45 \\
8 & 10.40 & 14.25 \\
9 & 10.11 & 23.84 \\
10 & 20.06 & 26.15 \\
11 & 10.11 & 23.85 \\
12 & 20.06 & 28.25 \\
13 & 11.68 & 15.52 \\
14 & 15.36 & 18.68 \\
15 & 6.93 & 14.24 \\
$16^{\mathrm{a}}$ & 12.88 & 11.04 \\
$17^{\mathrm{a}}$ & 17.35 & 13.6 \\
Mean & 13.19 & 18.63 \\
SD & 3.9 & 6.3 \\
\hline
\end{tabular}

Condition 1: reading dialogue at normal volume to another person standing at $1.5 \mathrm{~m}$. Condition 3: reading dialogue at loud volume to another person standing at $4 \mathrm{~m}$.

${ }^{a}$ Discordant subjects that did not fit the working hypothesis.

The mean VCOP rms was $7.25 \mathrm{~mm} / \mathrm{s}$ when the subject was empty-handed versus $12.77 \mathrm{~mm} / \mathrm{s}$ when the subject was holding a $3-\mathrm{kg}$ weight. This difference was statistically significant $(\mathrm{p}=0.003)$. An increase in VCOP rms was observed in all subjects during condition 2 .

Folia Phoniatr Logop 2008;60:80-85 


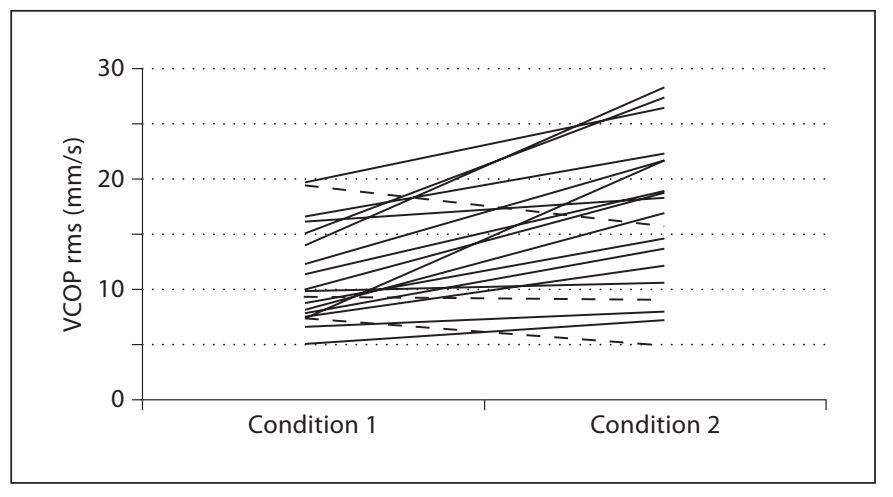

Fig. 4. Protocol 4: VCOP rms on y-axis. Condition 1: reading dialogue in calm surroundings. Condition 2: reading dialogue in noisy surroundings $(90 \mathrm{db})$. Stippled lines correspond to discordances with working hypothesis.

Table 4. Protocol 4: VCOP rms (in millimeters/second) values in different noise surroundings

\begin{tabular}{lrr}
\hline Subject & Condition 1 & Condition 2 \\
\hline 1 & 11.42 & 18.98 \\
2 & 6.55 & 8.01 \\
$3^{\mathrm{a}}$ & 9.35 & 8.94 \\
4 & 7.80 & 13.70 \\
5 & 7.40 & 21.75 \\
$6^{\mathrm{a}}$ & 7.52 & 5.02 \\
7 & 16.67 & 22.25 \\
8 & 16.08 & 18.24 \\
9 & 15.10 & 27.45 \\
10 & 10.05 & 18.75 \\
11 & 5.06 & 7.30 \\
12 & 12.25 & 21.65 \\
13 & 14.07 & 28.30 \\
14 & 8.71 & 14.65 \\
$15^{\mathrm{a}}$ & 19.45 & 15.65 \\
16 & 7.56 & 12.15 \\
17 & 9.80 & 10.57 \\
18 & 19.66 & 26.52 \\
19 & 8.15 & 16.85 \\
Mean & 11.19 & 16.67 \\
SD & 4.42 & 6.96 \\
\hline
\end{tabular}

Condition 1: calm ambiance. Condition 2: Lombard conditions.

${ }^{a}$ Discordant subjects that did not fit the working hypothesis.

Protocol 3 data are presented in table 3 and figure 3. The mean VCOP rms was significantly higher $(\mathrm{p}=0.017)$ during loud volume phonation (condition 2) than normal volume phonation (condition 1): 18.63 versus $13.19 \mathrm{~mm} / \mathrm{s}$. An increase in VCOP rms was observed in 13 of the 17 subjects tested. Discordance with the working hypothesis, i.e. no change during condition 2 , was observed in 4 subjects.

Protocol 4 data are presented in table 4 and figure 4 . VCOP rms was higher when the subjects performed under Lombard conditions than in a calm ambiance: 16.67 versus $11.19 \mathrm{~mm} / \mathrm{s}$. This difference was statistically significant $(\mathrm{p}=0.012)$. Discordance with the working hypothesis, i.e. no change in condition 2 , was observed in 3 subjects.

\section{Discussion}

This study confirms the utility of VCOP as an indicator of postural muscle stiffening during phonation. An increase in VCOP rms was associated with stiffening of the posture muscles induced by standing upright with the eyes closed (protocol 1) and holding a 3 -kg weight (protocol 2). Higher values were also related to a vocal effort in normal subjects speaking loudly to be understood at long distance (protocol 3) and under Lombard conditions (protocol 4).

Protocols 1 and 2 confirmed that VCOP rms could be correlated with muscle activity regardless of the amount of backward or forward body movement [16-20]. They also suggested that VCOP rms could be used for the indirect evaluation of postural muscle stiffening induced by the vocal forcing. Protocols 3 and 4 confirmed this hypothesis. The increase in vocal intensity is correlated with the VCOP rms, which has been shown to be an indicator of the postural muscular activity.

This study has several limitations. The precise acoustic level produced during protocols 3 and 4 was not determined. The only requirement for test subjects in condition 2 of protocol 3 was to be understandable at a distance of $4 \mathrm{~m}$. Similarly in condition 2 of protocol 4 (Lombard effect), the subjects received no specific instructions about vocal intensity. The protocols were designed to allow subjects to 'adjust' voice intensity as spontaneously as possible. For the same reason the microphone was used only to measure phonation time and to synchronize postural recordings. Since the microphone was not calibrated, it was not possible to analyze the correlation between VCOP rms and actual voice intensity. The goal of this study, i.e. correlating VCOP rms with stiffening of the body during vocal effort, did not require accurate determination of vocal intensity. Further study will be needed to clarify this point. 
Further study will also be required to explain discordances with the working hypotheses observed in protocols 3 and 4 . Two hypotheses can be formulated. The first is that some subjects used a different postural strategy when increasing acoustic intensity, e.g. improvement of postural stability to avoid instability. The second hypothesis is that failure of the discordant subjects to properly execute the experimental task, i.e. reading the text of the dialogue in realistic intensity conditions. In this regard, it can be speculated that the subjects did not raise their voice to the forcing level in condition 2 of protocols 3 and 4 or that they decreased the vocal effort as soon as they felt straining. Due to the absence of emotional content in the dialogue, it is also possible that the subjects had no interest in being understandable in the simulated cock- tail party surroundings. It would be useful to verify a dialogue with a more realistic 'emotional content' such as that occurring in a business setting.

\section{Conclusion}

The results of this study suggest that VCOP rms would be useful as a postural index for noninvasive assessment of the voice in pathology. However, several questions remain unanswered and further study will be needed using more realistic forcing conditions. To gain insight into clinical application, we have already undertaken a study on patients with muscular tension dysphonia and/or nodules.

\section{References}

-1 Kooijman PG, DeJong FI, Oudes MJ, Huinck W, Van Acht H, Graamans K: Muscular ten sion and body posture in relation to voice handicap and voice quality in teachers with persistent voice complaints. Folia Phoniatr Logop 2005;57:134-147.

2 Quarrier NF: Forward head posture in vocal performance. Med Probl Perform Art 1993; 17:29-32.

3 Gould WJ: Effect of respiratory and postural mechanisms upon action of the vocal cords. Folia Phoniatr 1971;23:211-224.

4 Le Huche F: La voix. Anatomie et physiologie des organes de la voix et de la parole. Paris, Masson, 1984.

5 Grini-Grandval MN, Ouaknine M, Giovanni A: Forcing the voice and variance of speed: correlation between the speed of displacement of the center of gravity and the work of postural muscles. Rev Laryngol Otol Rhinol 2000;121:319-323.

6 Bizzo G, Guillet N, Patat A, Gagey PM: Specification for building a vertical force platform designed for clinical stabilometry. Med Biol Eng Comput 1985;23:474-476.
7 Collins JJ, De Luca CJ: Random walking during quiet standing. Phys Rev Lett 1994;73: 764-767.

8 Lacour M, Barthélemy J, Borel L, Magnan J, Xerri C, Chays A, Ouaknine M: Sensory strategy in human postural control before and after unilateral vestibular neurotomy. Exp Brain Res 1997;115:300-310.

9 Crowe A, Schiereck P, de Boer W, Keessen W: Characterization of human gait by means of body center of mass oscillations derived from ground reaction forces. IEEE Trans Biomed Eng 1995;42:293-303.

10 Stockwell CW, Koozekanani SH, Barin K: A physical model of human postural dynamics. Ann NY Acad Sci 1981;374:722-730.

11 Yang JF, Winter AD, Wells RP: Postural dynamics in the standing human. Biol Cybern 1990;62:309-320.

12 SPSS Inc: Systat 6.0 for Windows. Chicago, SPSS Inc, 1996.

13 Lane H, Tranel B: The Lombard sign and the role of hearing in speech. J Speech Hear Res 1971;14:677-704.

14 Lamprecht A: Changes of voice in the Lombard reflex. Laryngol Rhinol Otol (Stuttg) $1988 ; 67: 350-354$
15 Ho AK, Bradshaw JL, Iansek R, Alfredson R: Speech volume regulation in Parkinson's disease: effects of implicit cues and explicit instructions. Neuropsychologia 1999;37: 1453-1460.

16 Alexandrov A, Frolov A, Massion J: Axial synergies during human upper trunk bending. Exp Brain Res 1998;118:210-220.

17 Fitzpatrick RC, Taylor JN, McCloskey DI: Ankle stiffness of standing humans in response to imperceptible perturbation: reflex and task-dependent components. J Physiol 1992;454:533-547.

18 Crenna P, Frigo C, Massion J, Pedotti A: Forward and backward axial synergies in man. Exp Brain Res 1987;65:538-548.

19 Winter DA, Prince F, Patla A: Validity of the invertum pendulum model of balance in quiet standing. Gait Posture 1997;5:153154.

20 McFarland DH, Smith A: Effects of task and respiratory phase on prephonatory chest wall movements. J Speech Hear Res 1992;35: 971-982. 